Journal of Computer Science 7 (4): 499-504, 2011

ISSN 1549-3636

(C) 2011 Science Publications

\title{
Object Exploration Using a Three-Axis Tactile Sensing Information
}

\author{
${ }^{1,2}$ S.C. Abdullah, ${ }^{1}$ Jiro Wada, ${ }^{1}$ Masahiro Ohka and ${ }^{1,2}$ Hanafiah Yussof \\ ${ }^{1}$ Graduate School of Information Science, Nagoya University Furo-cho Chikusa-ku, \\ Nagoya, Aichi, Japan \\ ${ }^{2}$ Faculty of Mechanical Engineering University Technology MARA, Malaysia
}

\begin{abstract}
Problem statement: To advance the robust object recognition of robots, we present an algorithm for object exploration based on three-axis tactile data that is necessary and sufficient for the evaluation of contact phenomena. Approach: The object surface contour is acquired by controlling the finger position so that the normal force, measured by optical three-axis tactile sensors, remains constant as two fingertips slide along the object surface. In this algorithm, when the robot grasps an object, the tangential force increment is checked to judge the initial contact state because it is more sensitive than the normal force. After contact between the fingertips and the object, the normal force is adjusted to remain constant with a tolerant value between the upper and lower thresholds. Results: In the verification test, shape exploration experiments were conducted using a hand-arm robot equipped with our tactile sensor and a hard sinusoidal-shaped wooden object. Experimental results show that the hand-arm robot is capable of gathering the object contour having a concave or convex portion because its finger position controlled by three-axis tactile sensing information follows the object surface. Conclusion/Recommendations: We derive a control algorithm in robot fingers based on time tangential force increment and normal force detection to perform a shape exploration procedure.
\end{abstract}

Key words: Object exploration, tactile-force sensing, three-axis, tactile sensor, object shape, normal force, tangential increment

\section{INTRODUCTION}

The requirements for autonomous service robots are the ability to detect, explore and recognize objects in their environments. Their modalities of applications highly differ from conventional industrial robots since they are deployed in a domestic environment and are usually not intended for repetitive tasks that require high precision or strong force. For such purposes, when a service robot must be equipped with sensors, vision sensors are popular choices. However, they may have limitations in such environmental conditions as in darkness, in very dirty or dusty situations, in foggy conditions, or even underwater. Being equipped with tactile sensors in addition to vision sensors is a good choice because the information of object properties is directly provided without influence of the environmental condition. Tactile sensors also offer great potential for improving the grasp synthesis in robot recognition and manipulation due to their extreme sensitivity and capability of measuring contact forces distribution.

We produced a unique tactile sensor system based on an optical waveguide transduction method for an application to humanoid robot hands (Ohka et al. 2004; 2005 and Yussof et al. 2010). The optical waveguide sensing principle used in this tactile sensor provides comparatively better sensing accuracy to detect contact phenomena from the acquisition of the three-axial directions of forces (Yussof et al. 2007).

Therefore it can simultaneously measure both normal and tangential forces and is suitable for robotic hand applications. For the development and the analysis of the control algorithm, the optical three-axis tactile sensors were mounted on the fingertips of a humanoid robot arm (Fig. 1) that consists of 11-dofs: 2-dofs at the shoulder joint, 1-dof at the elbow joint, 2-dofs at the wrist joint and 2 units of 3-dofs robot hands functioned as fingers.

In this study, we present an algorithm for obtaining the necessary and sufficient sensing information for object exploration procedures. In this algorithm, when the robot grasps an object, the tangential force increment is checked to determine the initial contact state because it is more sensitive than the normal force. After contact between the fingertips and the object, the normal force is adjusted to remain constant with a tolerant value between the upper and lower thresholds. In verification tests, shape exploration experiments were conducted using hard, sinusoidal-shaped wooden objects.

Corresponding Author: S.C. Abdullah, Graduate School of Information Science, Nagoya University, Furo-cho, Chikusa-ku, Nagoya, Aichi, Japan Tel / Fax: +81-52-789-4251 


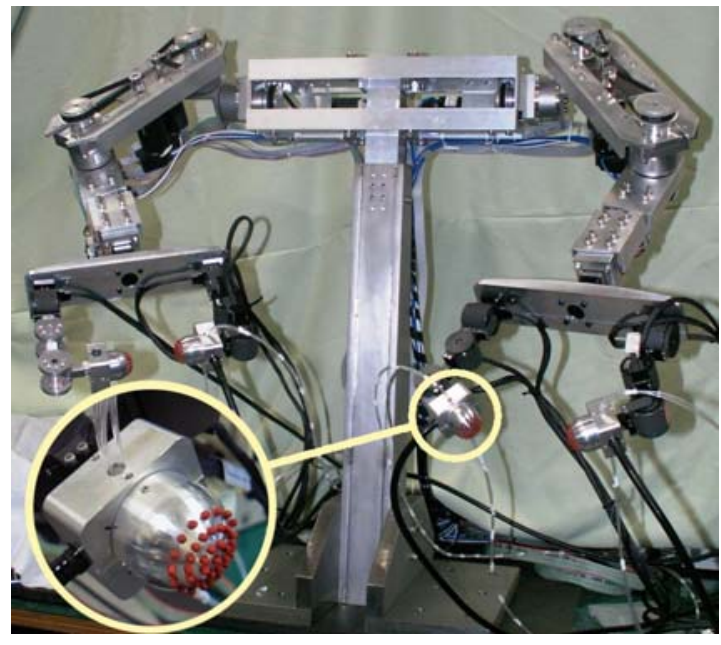

Fig. 1: 11-dof multi-fingered humanoid arm mounted with optical three-axis tactile sensors at fingertips

Related works: Object exploration is a typical human ability that is widely studied from both biological and engineering points of view. Indeed, identifying objects and collecting grasp and manipulation information that is as precise and accurate as human performance is crucial. Previous researches (Kaneko et al. 1992, Maekawa et al. 1992) mounted tactile sensors on robotic hands to evaluate grasping abilities and manipulation performance. Many studies developed procedures to determine the texture, hardness, shape and size of an object by haptic exploration. Other research (Klatzky et al. 1987) identified exploration procedures from observing such human exploration behavior as lateral motion, pressure, enclosure and contour following. Ali et al. (2010) in their research studying relationship between the forces and the deformation of the tactile sensor for alternative tactile sensor in future.

On the other hand (Russell and Wijaya, 2005), insect manners were mimicked by mounting whisker sensors on a mobile robot to perform object exploration. The whiskers are attached to a potentiometer to measure the angle of rotation when they touch an object. The sensitivity of a force as low as $5 \mathrm{mN}$ with high robustness and linked to visual recognition was proposed by Schmidt et al. (2006). Their sensor was equipped with grippers that consist of brushes of fibers. Moreover the development of tactile sensor matrices (Chen et al. 1995; Heidemann and Schoepfer, 2004) for edge tracking and object identification dynamically continues. In Okamura et al. (2001), contact trajectory information obtained from a tactile sensor is used to identify features while moving over and around them.
In conjunction with the above studies, our research has focused on the development of high performance tactile sensing devices for robotic hand applications because there is no three-axis tactile sensor outside of our research (Ohka et al. 2006 and Yussof et al. 2008a; $2008 b)$. In our previous works, we found that the time derivative of tangential force is very sensitive compared to normal force to detect contact between the sensor and the object. In our algorithm we apply the characteristics of tangential force to object exploration.

\section{MATERIALS AND METHODS}

Optical three-axis tactile sensor design: The design of our optical three-axis tactile sensor was motivated by the structure of human fingertips for easy compliance with various shaped objects (Fig. 2). Its system layout consists of an acrylic hemispherical dome, an array of 41 sensing elements made from silicon rubber, a light source, an optical fiber scope and a CCD camera.

When contact pressure is applied on the tactile sensor elements, a bright spot area appears inside the tactile sensor, which is then captured as image data by a CCD camera. The image data retrieved by the CCD camera are delivered to a PC by a PCI bus of a Himawari PCI/S image processing board. The image data are saved in an internal buffer area that is created inside the PC internal memory space. The control system architecture (Fig. 3) of the robot hand is comprised of three modules: a Connection Module, Thinking Routines and a Hand Control Module. The architecture is connected to the tactile sensor controller by the connection modules using TCP/IP. The details of this system layout were explained in Yussof et al. 2008a.

Based on this relationship, Yussof (et al. 2008a) defined the normal force from the gray-scale distribution $\mathrm{g}(\mathrm{x}, \mathrm{y})$ of contact area $S$ caused by contact between a sensing element and an acrylic dome based on the following equation:

$P=C_{v} \int_{S} g(x, y) d S$

Where $\mathrm{C}_{\mathrm{v}}$ is a constant decided by calibration tests.

The tangential force components of applied force vectors are obtained from movement of the contact area centroid.

Object exploration algorithm: Previous study (Yussof et al. 2008a; 2008b) discussed suitable tactile sensingbased control parameters in our robot arm control system using the optical three-axis tactile sensor. In this 



Fig. 2: Design of optical three-axis tactile sensor

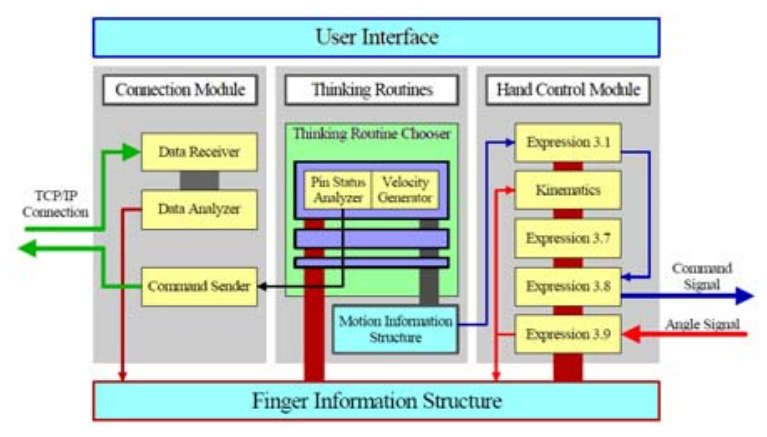

Fig. 3: Control system structure of robot

research we defined new tactile sensing-based control parameters for better and smoother object shape exploration. The robot control parameters are shown in Table 1. We proposed a control algorithm to distinguish object shape and to generate suitable grasp motion for exploration along the object surface hand.
Table 1 Parameters of stiffness control

\begin{tabular}{lll}
\hline Category & & Parameter \\
\hline Sampling interval & Sensor & $100 \mathrm{~ms}$ \\
& Finger & $25 \mathrm{~ms}$ \\
Threshold of normal force & $\mathrm{F}_{1}$ & $0.08 \mathrm{~N}$ \\
& $\mathrm{~F}_{2}$ & $0.2 \mathrm{~N}$ \\
Threshold of tangential force & $\mathrm{dr}$ & 0.004 \\
mm/step & & \\
difference during two steps & & $1 \mathrm{~mm} / \mathrm{s}$ \\
Velocity of re-push & $\mathrm{v}_{\mathrm{p}}$ & \\
\hline
\end{tabular}

We classified touch movements into three classifications to control the finger-grasping pressure for shape exploration: finger-close, finger-hold and finger-open. These classifications are used to select the correct movement for the velocity of re-push motion $v_{p}$. The threshold of normal forces $F_{1}$ and $F_{2}$ control the finger movements during grasping and exploring object shape.

The experiment used robot fingers and optical three-axis tactile sensors that were mounted on the fingertips. To obtain normal force distribution from our tactile sensor a series of experiments explored real object surfaces. Motion planning was designed so that both fingers could move along the $x$-axis direction to explore the object.

The experiment conditions are shown in Fig. 4. In the $x$-axis direction, the robot fingers explored a curved sinusoidal-shaped wooden object that was $285 \mathrm{~mm}$ high and $30 \mathrm{~mm}$ wide with three convex and two concave parts on both sides (Fig. 5). The robot arm moves its fingers to explore the shape surface at $0.5 \mathrm{~mm} / \mathrm{s}$.

Figure 6 shows the algorithm flowchart for object shape exploration using a robotic finger equipped with an optical three-axis tactile sensor. In this algorithm, two major blocks, "Touch" and "Grasping Force Adjustment," are sequentially connected. First, the robotic hand searches for the object surface by moving its fingers toward the object surface. In the "Touch" block, tangential force increment $d F_{t}$ judges whether the fingertip touched the object. If the tangential force increment exceeds threshold $d r$, shown in Table 1, it judges that contact has occurred. After that, grasping force adjustment is performed in the proposed control system by recognizing the response to the changes of object shape during the object exploring tasks. In the control system, the fingertip velocity is determined based on the three flag values. Detailed explanations are as follows:

- First, in the "Touch" block, both fingers softly touch the object based on the judgment of the tangential force increment

- After "Touch," the $F_{1}$ and $F_{2}$ parameters are used to control the grip force 


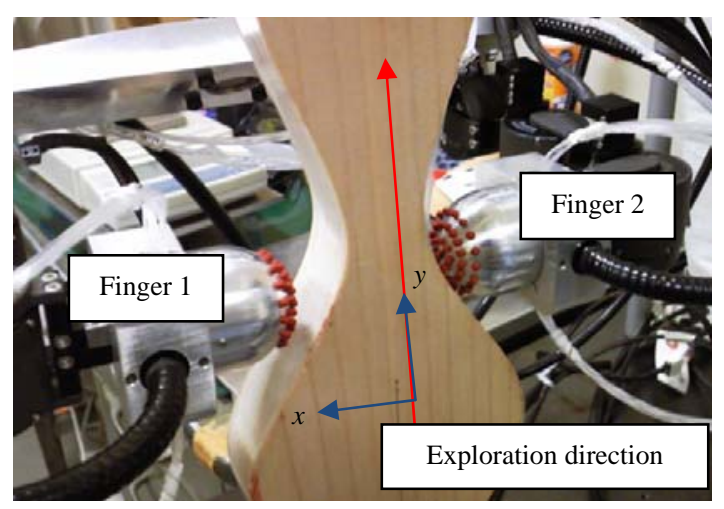

Fig. 4 Object shape exploration

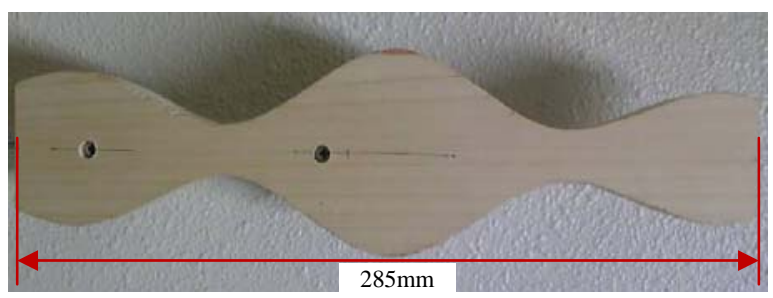

Fig. 5 Object used in experiment

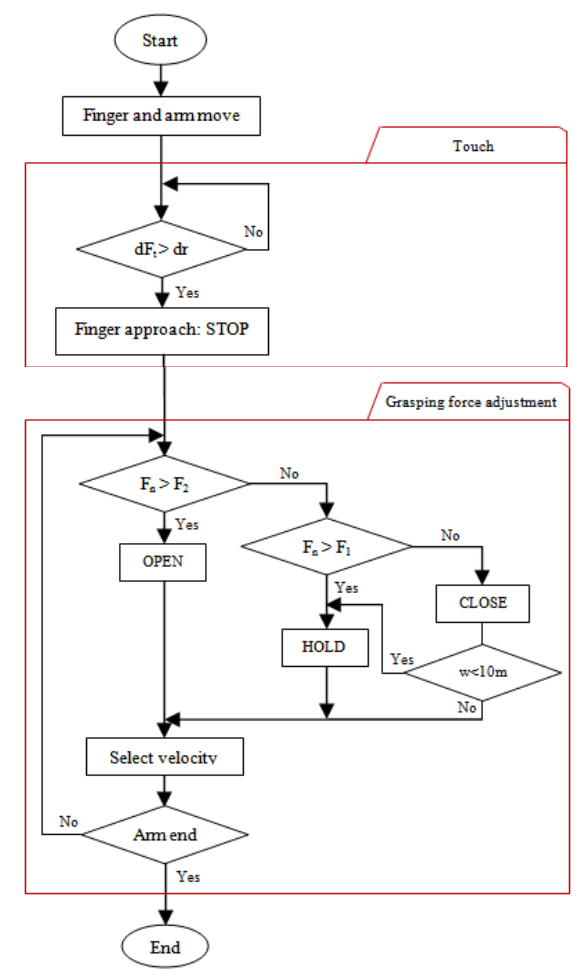

Fig. 6: Algorithm flowchart of touch motion robot fingers
- $\quad$ During the exploring motion, if normal force $F_{n}[N]$ that exceeds threshold $F_{2}[\mathrm{~N}]$ OPEN flag is raised, the fingers will open

- If the $F_{n}[N]$ does not exceed $F_{2}[N]$, but the exceeding $\mathrm{F}_{1}[\mathrm{~N}]$ HOLD flag is raised, the fingers will hold the movement and move toward the $\mathrm{x}$ axis of the object surface until they reach the new threshold

- If $F_{n}[N]$ does not exceed $F_{1}[N]$, the CLOSE flag continues until it reaches a minimum width at 10 $\mathrm{mm}$ at which the fingers stop moving. Otherwise the fingers will continue to close until they reach a new threshold

- Then the list of data is sent to the connection module, which receives the fedback normal force data and sends them to the finger information structure. Based on this data, the velocity generator module decides the finger velocity

\section{RESULTS}

We conducted a series of experiments using the two robotic fingers to explore real objects to obtain normal force distribution from our tactile sensor. The normal force distribution is performed in the proposed control system by recognizing the response to the changes of object shape during the object exploring tasks.

In the experiment result (Fig. 7), the black line is the $x$-axis direction of the finger position and the dots are the normal force distribution represented by 17 sensor elements of the optical three-axis tactile sensor.

Figure 7(a) shows that the start position of normal force distribution position finger 1 was at $30.34 \mathrm{~m}$ and it moves down to $12.72 \mathrm{~mm}$ around 58,031 msec. Then it goes up to $26.45 \mathrm{~mm}$ at $165,831 \mathrm{msec}$. Fig. 7(b) shows the other way around: the start position at -30.33 $\mathrm{mm}$ and it moves up to $-10.99 \mathrm{~mm}$ at $58,906 \mathrm{msec}$. Then it goes down to reach $-24.54 \mathrm{~mm}$ at $165,831 \mathrm{msec}$. These two figures show that the movement of exploration starts at a wide convex position, moves to a narrow concave positio and then moves toward the next convex position again. From variations in normal force represented by dots, the sensing elements measuring higher values are alternated at the concave portion (around 60,000 msec). At this point, the sensor's contact point is changed because this object exploring motion is performed under a fixed attitude of the finger. 




(a)

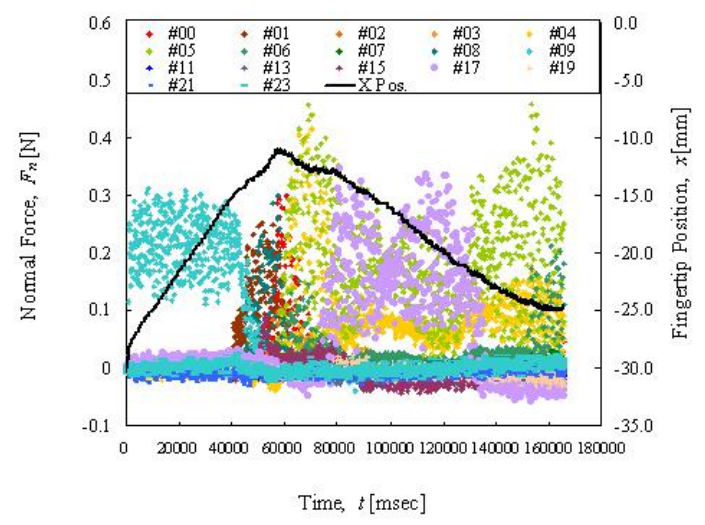

(b)

Fig. 7: Relationships between normal force accepted by sensing elements and time spent and between $\mathrm{x}$ directional position of finger and time spent

\section{DISCUSSION}

Normal force distribution shows which sensing element maintains contact with the object surface during the exploration experiment and can be distinguished by the representation of 17 sensing elements in each finger. The concave peak of the variation in the normal force becomes higher than that before the concave peak. The normal force of the representative sensing element varies in the range of $0.38-0.60 \mathrm{~N}$ at finger 1 and $0.32-0.47 \mathrm{~N}$ at finger 2 when the fingers move from the concave peak $(60,000$ msec) to the convex peak $(165,931 \mathrm{msec})$ and it varies in the range of $0.25-0.42 \mathrm{~N}$ and $0.28-0.31 \mathrm{~N}$ for each finger when the fingers start exploration from the convex peak ( $0 \mathrm{msec})$ to the concave $(60,000 \mathrm{msec})$ position. This situation occurs due to the different working thresholds before and after the concave peak.
From the plotted graphs of the $x$ directional position (the local $x$ coordinate is defined as the hand open/close direction (Fig. 4), the contour of the object surface can be obtained because the black line traces the cosine-like curve of the specimen profile. Therefore our robotic hand, which is equipped with optical three-axis tactile sensors and the present exploration algorithm, can extract an object's contour having convex and concave portions.

\section{CONCLUSION}

In the present study, we derive a control algorithm in robot fingers based on tangential force increment and normal force to perform a shape exploration task. The fingertips of the robot fingers are equipped with optical three-axis tactile sensors. To evaluate the shape exploration algorithm, we conducted several experiments of path-guided object-grasp touching exploration with the robotic fingers. The experimental results revealed that the wavy aspect of the object shape was acquired by the differences in the normal force distribution along the path of the object exploration. The finger positions show that the robot control system responded to the wavy object shape and the results revealed good performance for the robot fingers in defining the optimum grasp pressure and in autonomously controlling the grasp and release motions for object shape exploration at an arbitrary position.

We expect that this tactile sensing technology control scheme will advance the evolution of robotic object handling tasks in unknown environments to replace humans in tedious or dangerous work. In our next study, we will discuss the novel algorithm that combines the tangential and normal forces; in our present algorithm, tangential force and normal force blocks are connected sequentially. Further study on the affect of coefficient of friction $(\mu)$ on exploration behavior will be carried out in future research.

\section{REFERENCES}

Ali, B., R. Othman, R. Deraman and M.A. Ayub, 2010. A new approach in design and operating principle of silicone tactile sensor. J. Comput. Sci., 6: 940-945. DOI: $10.3844 /$ jcssp.2010.940.945

Chen, N., H. Zhang and R. Rink, 1995. Edge tracking using tactile servo. Proceedings of the International Conference on Intelligent Robots and Systems 95. Human Robots Interaction and Cooperative Robots, Aug. 5-9, IEEE Xplore, Pittsburgh, PA, USA., pp: 84-89. DOI: 10.1109/IROS.1995.526143 
Heidemann, G. and M. Schoepfer, 2004. Dynamic tactile sensing for object identification. Proceedings of the IEEE International Conference Robotics and Automation ICRA 2004, Apr. 26-1 May, Bielefeld University, Germany, pp: 813-818. DOI: 10.1109/ROBOT.2004.1307249

Kaneko, M., H. Maekawa and K. Tanie, 1992. Active tactile sensing by robotic fingers based on minimum-external-sensor-realization. Proceeding of the IEEE International Conference on Robotics and Automation ICRA92, May 12-14, IEEE Xplore, Nice, France, pp: 1289-1294. DOI: 10.1109/ROBOT.1992.220071

Klatzky, R.L., S.J. Lederman and C. Reed, 1987. There's more to touch than meets the eye: The salience of object attributes for haptics with and without vision. J. Exp. Psychol.: General, 116: 356-369. DOI: 10.1037/0096-3445.116.4.356

Maekawa, H., K. Tanie, K. Komoriya, M. Kaneko and C. Horiguchi et al., 1992. Development of a fingershaped tactile sensor and its evaluation by active touch. Proceedings of the IEEE Int. Conf. on Robotics and Automation, May 12-14, IEEE Xplore, Nice, France, pp: 1327-1334. DOI: 10.1109/ROBOT.1992.220165

Ohka, M., H. Kobayashi and Y. Mitsuya, 2005. Sensing characteristics of an optical three-axis tactile sensor mounted on a multi-fingered robotic hand. Proceedings of the International Conference on Intelligent Robots and Systems, Aug. 2-6, Nagoya University, Japan, pp: 493-498. DOI: 10.1109/IROS.2005.1545264

Ohka, M., H. Kobayashi and Y. Mitsuya, 2006. Sensing precision of an optical three-axis tactile sensor for a robotic finger. Proceedings of 15th IEEE International Symposium on Robot and Human Interactive Communication, Sept. 6-8, IEEE Xplore, Hatfield, pp: 214-219. DOI: 10.1109/ROMAN.2006.314420

Ohka, M., Y. Mitsuya, Y. Matsunaga and S. Takeuchi, 2004. Sensing characteristics of an optical threeaxis tactile sensor under combined loading. Robotica, 22: 213-221. DOI: 10.1017/S0263574703005538
Okamura, A.M. and M.R. Cutkosky, 2001. Feature detection for haptic exploration with robotic fingers. Int. J. Robotics Res. 20: 925-938. DOI: 10.1177/02783640122068191

Russell, R.A. and J.A. Wijaya, 2005. Recognising and Manipulating Objects Using Data From a Whisker Sensor Array. Robotica, 23: 653-664. DOI: 10.1017/S0263574704000748

Schmidt, P.A., E. Maël and R.P. Würtz, 2006. A sensor for dynamic tactile information with applications in human-robot interaction and object exploration. Robot. Auto. Syst., 54: 1005-1014. DOI: 10.1016/j.robot.2006.05.013

Yussof, H., J. Wada and M. Ohka, 2010. Sensorization of robotic hand using optical three-axis tactile sensor: Evaluation with grasping and twisting motions. J. Comput. Sci., 6: 955-962. DOI: 10.3844/jcssp.2010.955.962

Yussof, H., M. Ohka, A.R. Omar and M.A. Ayub, 2008a. Determination of object stiffness control parameters in robot manipulation using a prototype optical three-axis tactile sensor. Proceeding of The 7th IEEE International Conference on Sensors (SENSORS2008), Oct. 26-29, IEEE Xplore, Lecce, pp: 992-995. DOI: 10.1109/ICSENS.2008.4716609

Yussof, H., M. Ohka, H. Kobayashi, J. Takata, M. Yamano and Y. Nasu, 2007. Development of an optical three-axis tactile sensor for object handing tasks in humanoid robot navigation system. Autonomous Robo. Agents, 76: 43-51. DOI: 10.1007/978-3-540-73424-6_6

Yussof, H., M. Ohka, J. Takata, Y. Nasu and M. Yamano, 2008b. Low force control scheme for object hardness distinction in robot manipulation based on tactile sensing. Proceedings of The 2008 IEEE International Conference on Robotics and Automation, May 19-23, IEEE Xplore, Pasadens, USA., $\quad$ pp: 3443-3448. DOI: 10.1109/ROBOT.2008.4543737 